\title{
Clinical and Imaging Characteristics of Diffuse Intracranial Dolichoectasia
}

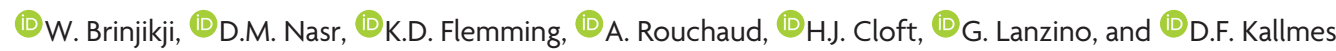

\begin{abstract}
BACKGROUND AND PURPOSE: Among patients with vertebrobasilar dolichoectasia is a subset of patients with disease affecting the anterior circulation as well. We hypothesized that multivessel intracranial dolichoectasia may represent a distinct phenotype from single-territory vertebrobasilar dolichoectasia. The purpose of this study was to characterize clinical characteristics and angiographic features of this proposed distinct phenotype termed "diffuse intracranial dolichoectasia" and compare them with those in patients with isolated vertebrobasilar dolichoectasia.
\end{abstract}

MATERIALS AND METHODS: We retrospectively reviewed a consecutive series of patients with diffuse intracranial dolichoectasia and compared their demographics, vascular risk factors, additional aneurysm prevalence, and clinical outcomes with a group of patients with vertebrobasilar dolichoectasia. "Diffuse intracranial dolichoectasia" was defined as aneurysmal dilation of entire vascular segments involving $\geq 2$ intracranial vascular beds. Categoric and continuous variables were compared by using $\chi^{2}$ and Student $t$ tests, respectively.

RESULTS: Twenty-five patients had diffuse intracranial dolichoectasia, and 139 had vertebrobasilar dolichoectasia. Patients with diffuse intracranial dolichoectasia were older than those with vertebrobasilar dolichoectasia $(70.9 \pm 14.2$ years versus $60.4 \pm 12.5$ years, $P=.0002)$ and had a higher prevalence of abdominal aortic aneurysms (62.5\% versus $14.3 \%, P=.01)$, other visceral aneurysms $(25.0 \%$ versus $0 \%, P<.0001)$, and smoking $(68.0 \%$ versus $15.9 \%, P<.0001)$. Patients with diffuse intracranial dolichoectasia were more likely to have aneurysm growth $(46.2 \%$ versus $21.5 \%, P=$ .09 ) and rupture (20\% versus $3.5 \%, P=.007)$ at follow-up. Patients with diffuse intracranial dolichoectasia were less likely to have good neurologic function at follow-up $(24.0 \%$ versus $57.6 \%, P=.004)$ and were more likely to have aneurysm-related death $(24.0 \%$ versus $7.2 \%, P=.02)$.

CONCLUSIONS: The natural history of patients with diffuse intracranial dolichoectasia is significantly worse than that in those with isolated vertebrobasilar dolichoectasia. Many patients with diffuse intracranial dolichoectasia had additional saccular and abdominal aortic aneurysms. These findings suggest that diffuse intracranial dolichoectasia may be a distinct vascular phenotype secondary to a systemic arteriopathy affecting multiple vascular beds.

$\mathbf{F}$ usiform and dolichoectatic intracranial aneurysms are generally associated with a poor natural history with high rates of rupture, mass effect, and ischemic stroke. ${ }^{1-4}$ These aneurysms often form secondary to circumferential loss or weakening of the internal elastic lamina of a vessel segment and can involve an entire vessel or may be limited to a single vessel segment (eg, basilar artery, cavernous or supraclinoid ICA, proximal MCA, and so forth). ${ }^{5-8}$ Notably, most prior reports of intracranial

Received August 12, 2016; accepted after revision December 13

From the Departments of Radiology (W.B., H.J.C., G.L., D.F.K.), Neurology (D.M.N., K.D.F., G.L.), and Neurosurgery (W.B., H.J.C., G.L., D.F.K.), Mayo Clinic, Rochester, Minnesota, and Department of Interventional Neuroradiology (A.R.), Hôpital Bicêtre, Paris Sud Universite, Paris, France.

Please address correspondence to Waleed Brinjikji, MD. Mayo Clinic, 200 1st St SW, Rochester, MN 55905; e-mail: brinjikji.waleed@mayo.edu; @wbrinjikji

三 Indicates article with supplemental on-line table.

http://dx.doi.org/10.3174/ajnr.A5102 dolichoectasia have focused on involvement of single-vessel territories, most commonly the vertebrobasilar system. These dolichoectatic aneurysms are associated with a wide range of proposed, underlying etiologies, including healed, focal arterial dissections; chronic hypertension; prior radiation therapy; connective tissue diseases; arterial tortuosity syndrome; glycogen storage diseases; infection; and myxomatous emboli. ${ }^{5-8}$

During the past several years, we have noticed in our clinical practice a subset of patients who have segmental aneurysmal dolichoectasia of multiple intracranial vascular territories in the absence of a known, underlying insult such as radiation, infection, genetic connective tissue disease, or dissection. We hypothesized that multivessel presentation of intracranial dolichoectasia may represent a process distinct from the frequently described single-territory dolichoectasia, potentially with distinct presentation, associations, and natural history. For this report, we have defined "diffuse intracranial dolichoectasia" as aneurysmal dilation of entire segments that involve $\geq 2$ in- 


\begin{tabular}{llc} 
Vascular Segment & \multicolumn{1}{c}{ Definition of Vascular Segment } & $\begin{array}{c}\text { Definition of Aneurysmal } \\
\text { Dilation }\end{array}$ \\
\hline Cavernous ICA & Entry point of ICA into the cavernous segment to the ophthalmic artery origin & $\geq 8.5 \mathrm{~mm}$ \\
Supraclinoid ICA & ICA from the ophthalmic artery origin to the ICA bifurcation & $\geq 8.0 \mathrm{~mm}$ \\
M1 segment of MCA & Origin of MCA to the MCA bifurcation & $\geq 5.0 \mathrm{~mm}$ \\
Basilar artery & Confluence of vertebral arteries to the basilar bifurcation & $\geq 6.0 \mathrm{~mm}$ \\
\hline
\end{tabular}

tracranial vascular beds. These criteria were chosen due to the notion that fusiform aneurysmal dilation of multiple intracranial vascular beds indicates a systemic vascular insult, while fusiform aneurysmal dilation of a single bed suggests a more localized pathologic process.

The primary purpose of this article was to fully characterize the clinical risk factors and anatomic and angiographic characteristics of this proposed distinct entity, which we term "diffuse intracranial dolichoectasia." The secondary purpose was to compare clinical risk factors and anatomic and angiographic characteristics of patients with diffuse intracranial dolichoectasia with those in a group of patients with dolichoectasia isolated to the vertebrobasilar system.

\section{MATERIALS AND METHODS Patient Population}

Following institutional review board approval, we identified all patients with diffuse intracranial dolichoectasia evaluated at our institution from January 1, 2001, to December 31, 2014. We also identified a control group of patients with isolated vertebrobasilar dolichoectasia. To identify patients, we searched our imaging data base for reports of head MR imaging, MRA, CTA, and DSA examinations in which any one of the following terms was used in the body or conclusions of the report: "fusiform," "aneurysmal dilation," "dilation," "fusiform aneurysm," "dolichoectasia," "dolichoectatic," and "dolicho." All identified imaging examinations were then reviewed by 2 radiologists in consensus. Cases with fusiform aneurysmal dilation of multiple vascular segments were then identified for additional review to determine whether they met the diagnostic criteria for diffuse intracranial dolichoectasia. Cases in which there was fusiform aneurysmal dilation with or without tortuosity isolated to the vertebrobasilar system were defined as "isolated vertebrobasilar dolichoectasia." Of the cases that were identified for additional review as potentially representing diffuse intracranial dolichoectasia, maximum measurements were obtained of the basilar artery, supraclinoid ICAs, and bilateral M1 segments.

\section{Diagnostic Criteria}

To be included in this diffuse intracranial dolichoectasia group, patients had to meet the following diagnostic inclusion criteria: adult patients with fusiform aneurysmal dilation of entire vascular segments (ie, supraclinoid ICA, basilar artery, M1 segment of the MCA) that involved $\geq 2$ intracranial vascular beds (ie, vertebrobasilar system, left anterior circulation, or right anterior circulation). Aneurysmal dilation of a vascular segment was defined as a diameter of at least 2 times the normal diameter of a given vascular segment as defined by an angiographic atlas. ${ }^{9}$ Isolated vertebrobasilar dolichoectasia was defined as an aneurysmal dilation 2 times the normal diameter without a definable neck involving a portion of an arterial segment (either vertebral or basilar) with any degree of tortuosity. The criteria for aneurysmal dilation of a vessel segment are summarized in Table 1. Exclusion criteria included the following: 1) pediatric patients, 2) patients with underlying connective tissue diseases (ie, Ehlers-Danlos and Marfan syndromes, Loeys-Dietz syndrome, autosomal dominant polycystic kidney disease, neurofibromatosis type 1, and so forth), 3) patients with a history of cranial radiation, 4) patients with an underlying infectious etiology (ie, varicella zoster vasculopathy, HIV, and so forth), and 5) patients with poststenotic dilations secondary to atherosclerosis or dissection. All patients in the vertebrobasilar dolichoectasia control group and 11 patients in the diffuse intracranial dolichoectasia group were also included in a prior study on the imaging natural history of patients with vertebrobasilar, nonsaccular dolichoectatic aneurysms. ${ }^{10}$

\section{Baseline Demographics and Clinical Risk Factors}

We collected the following baseline patient demographic characteristics and comorbidities: age; sex; the presence of an ectatic abdominal aorta (maximum diameter, $2.6-2.9 \mathrm{~cm}$ ) or an abdominal aortic aneurysm (maximum diameter, $\geq 3 \mathrm{~cm}$ ); and the presence of other vascular ectasias/aneurysms, fibromuscular dysplasia, coronary artery disease, peripheral artery disease, hypertension, diabetes mellitus, hyperlipidemia, tobacco use (current, former, never), packyears of tobacco use (if available), alcohol abuse, sympathomimetic abuse, and family history of abdominal aortic aneurysm, stroke, or aneurysm. In addition to baseline demographic and clinical risk factors, we collected data on presenting symptoms at the time of the discovery of the intracranial dolichoectasia (ie, incidental, stroke, subarachnoid hemorrhage, neuralgia, mass effect, headache, hemifacial spasm, other). Baseline demographic and clinical risk factors were compared between the diffuse intracranial dolichoectasia group and the isolated vertebrobasilar dolichoectasia group.

\section{Imaging Characteristics}

Among patients who met the diagnostic criteria for diffuse intracranial dolichoectasia and those in the isolated vertebrobasilar dolichoectasia control group, we collected the following information from their imaging examinations: the presence of a saccular aneurysm; the presence of other intracranial vascular dilations, subarachnoid hemorrhage, or intraparenchymal hemorrhage on presentation; white matter ischemic disease; and thrombus within the aneurysm. All measurements on CTA and MRA were obtained by using multiplanar reformatting software (Terarecon, San Mateo, California). The maximum cross-sectional diameter of the vessel perpendicular to the longitudinal axis of the vessel was obtained. For DSA images, the maximum diameter of the vessel perpendicular to the longitudinal axis of the vessel was obtained. Imaging characteristics were compared between the diffuse intracranial dolichoectasia group and the isolated vertebrobasilar dolichoectasia group. 
Table 2: Summary of patient characteristics and outcomes

\begin{tabular}{|c|c|c|c|}
\hline Variable & DID & VBD & $P$ Value \\
\hline No. & 25 & 139 & - \\
\hline Mean age (SD) (yr) & 70.9 (14.2) & $60.4(12.5)$ & .0002 \\
\hline \multicolumn{4}{|l|}{ Age group (yr) } \\
\hline $20-29$ & $1(4.0)$ & $5(3.6)$ & $<.0001$ \\
\hline $30-39$ & $0(0.0)$ & $6(4.3)$ & \\
\hline $40-49$ & $1(4.0)$ & $6(4.3)$ & \\
\hline $50-59$ & $2(8.0)$ & $44(31.6)$ & \\
\hline $60-69$ & $7(28.0)$ & $43(30.9)$ & \\
\hline 70-79 & $6(24.0)$ & $30(21.5)$ & \\
\hline$\geq 80$ & $8(32.0)$ & $5(3.6)$ & \\
\hline \multicolumn{4}{|l|}{ Sex } \\
\hline Male & $21(84.0)$ & $105(75.5)$ & .51 \\
\hline Female & $4(16.0)$ & $34(24.5)$ & \\
\hline \multicolumn{4}{|l|}{ Indication for imaging } \\
\hline Stroke & $13(52.0)$ & $45(32.7)$ & .10 \\
\hline Mass effect & $5(20.0)$ & $25(18.0)$ & .81 \\
\hline Other/incidental & $7(28.0)$ & $69(49.6)$ & .08 \\
\hline \multicolumn{4}{|l|}{ Comorbidities } \\
\hline Abdominal aortic aneurysm or ectasia & $10(62.5)$ & $12(14.3)$ & .01 \\
\hline Other visceral aneurysms & $4(25.0)$ & $0(0.0)$ & $<.0001$ \\
\hline Fibromuscular dysplasia & $0(0.0)$ & $0(0.0)$ & 1.0 \\
\hline Coronary artery disease & $10(40.0)$ & $40(29.0)$ & .38 \\
\hline Peripheral artery disease & $4(16.0)$ & $6(4.3)$ & .07 \\
\hline Hypertension & $22(88.0)$ & $97(70.3)$ & .11 \\
\hline Diabetes mellitus & $3(12.0)$ & $22(15.9)$ & .85 \\
\hline Hyperlipidemia & $13(52.0)$ & $73(52.9)$ & .96 \\
\hline Smoking & $17(68.0)$ & $22(15.9)$ & $<.0001$ \\
\hline Alcohol abuse & $1(4.0)$ & $7(5.0)$ & .82 \\
\hline Sympathomimetic abuse & $0(0.0)$ & $0(0.0)$ & 1.0 \\
\hline \multicolumn{4}{|l|}{ Family history } \\
\hline Abdominal aortic aneurysm & $3(12.0)$ & $9(6.4)$ & .58 \\
\hline Ischemic stroke & $7(28.0)$ & $32(23.0)$ & .78 \\
\hline Intracranial aneurysm & $1(4.0)$ & $9(6.4)$ & .98 \\
\hline Other saccular aneurysms present & $7(28.0)$ & $22(15.8)$ & .24 \\
\hline Growth on follow-up & $6(46.2)$ & $30(21.6)$ & .09 \\
\hline SAH on follow-up & $5(20.0)$ & $5(3.5)$ & .007 \\
\hline Infarct on follow-up & $7(28.0)$ & $18(13.0)$ & .10 \\
\hline \multicolumn{4}{|l|}{ Clinical status at last follow-up } \\
\hline Good neurologic function & $6(24.0)$ & $80(57.6)$ & .004 \\
\hline Poor neurologic function & $6(24.0)$ & $22(15.8)$ & .48 \\
\hline Death & $13(52.0)$ & $37(26.6)$ & .02 \\
\hline Aneurysm-related death & $6(24.0)$ & $10(7.2)$ & .02 \\
\hline
\end{tabular}

Note:-VBD indicates vertebrobasilar dolichoectasia; DID, diffuse intracranial dolichoectasia. performed a multivariate logistic regression analysis, adjusting for baseline factors that were different between groups (ie, age and smoking history). All analysis was performed by using the SAS-based statistical software package JMP 12.0 (SAS Institute, Cary, North Carolina).

\section{RESULTS}

Baseline Patient Characteristics for Diffuse Intracranial Dolichoectasia

The search of radiology reports (ie, MR imaging, MRA, CTA, and DSA) yielded 890 unique patients with the terms "fusiform," "aneurysmal dilation," "dilation," "fusiform aneurysm," "dolichoectasia," "dolichoectatic," and "dolicho" in their reports. After further review of imaging reports and radiologic images, 25 patients met the diagnostic criteria for diffuse intracranial dolichoectasia.

The mean patient age was $70.9 \pm 14.2$ years. Two patients were younger than 50 years of age $(8.0 \%)$, and 21 patients $(84.0 \%)$ were 60 years of age or older. Eighty-four percent (21/25) of patients were men. The most common clinical presentation was acute ischemic stroke or transient ischemic attack $(13 / 25,52 \%)$. Of the ischemic strokes, 6 affected the posterior circulation and 7 affected the anterior circulation. Five patients (20\%) had symptoms due to mass effect from the aneurysmal dilations, 3 patients (12\%) had dizziness, and in the remaining 4 patients $(16 \%)$, the aneurysms were discovered incidentally during evaluation for loss of consciousness, syncope, or visual blurring.

Among patients with abdominal im-

\section{Outcomes Studied}

In cases in which there was serial imaging, we collected data on presence of dolichoectatic aneurysm growth or rupture and new ischemic stroke. We also collected data on the clinical status of the patient at last follow-up. Clinical status was defined as no or minimal disability ( $\mathrm{mRS} \leq 2$ ), moderate or severe disability (mRS 3-5), and death. Among patients who died, the cause of death was noted, when available. Outcomes were compared between the diffuse intracranial dolichoectasia group and the isolated vertebrobasilar dolichoectasia group.

\section{Statistical Analysis}

All categoric variables are reported as number (percentage), while continuous variables are reported as mean \pm SD. Categoric variables were compared by using $\chi^{2}$ tests, and continuous variables were compared by using a Student $t$ test. To determine whether a diagnosis of diffuse intracranial dolichoectasia was independently associated with aneurysm growth, rupture, and new infarct, we aging, 50\% (8/16) had an abdominal aortic aneurysm and an additional 13\% (2/16) had aortic ectasia. Hypertension was present in $88 \%(22 / 25)$ of patients, and $68 \%(17 / 25)$ were current or former smokers. Hyperlipidemia was present in $52 \%$ (13/25) of patients, and intracranial atherosclerosis was present in 48\% (12/ 25). These data are summarized in Table 2.

\section{Angiographic and Anatomic Characteristics for Diffuse Intracranial Dolichoectasia}

The most common distribution of disease was dilation of the basilar artery and 1 internal carotid artery (10 patients, 40\%). Five patients $(20 \%)$ had diffuse dilation of the basilar artery and bilateral ICAs, and 4 patients (16\%) had dilation of the bilateral ICAs without dilation of the basilar artery. Two patients (8.0\%) had dilation of the basilar artery and bilateral ICAs and MCAs.

The mean maximum diameter of the dilated basilar arteries was $15.2 \pm 8.3 \mathrm{~mm}$. The mean maximum diameter of the dilated 
ICA segments was $12.7 \pm 7.0 \mathrm{~mm}$, and the mean maximum diameter of dilated MCA segments was $10.1 \pm 4.3 \mathrm{~mm}$. Seven patients $(28 \%)$ had additional intracranial saccular aneurysms. These data are summarized in Table 3.

\section{Outcomes for Diffuse Intracranial Dolichoectasia}

Thirteen patients had imaging follow-up (mean, $54.1 \pm 78.4$ months). Of these patients, 6 (46\%) had growth of at least 1 aneurysmal dolichoectasia, which occurred at a mean of $56.0 \pm 54.3$ months. Given a total of 63.1 patient-years of imaging follow-up,

Table 3: Angiographic characteristics and distribution of DID

\begin{tabular}{lc}
\hline & No. (\%) \\
\hline Vessels involved & \\
Anterior circulation only & $4(16.0)$ \\
Bilateral ICAs & $4(16.0)$ \\
Anterior and posterior circulation & $21(84.0)$ \\
Basilar artery + 1 ICA & $10(40.0)$ \\
Basilar artery + bilateral ICAs & $5(20.0)$ \\
Basilar artery + bilateral ICAs + bilateral MCAs & $2(8.0)$ \\
Basilar artery + 1 ICA/ipsilateral M1 & $2(8.0)$ \\
Basilar artery + 1 ICA + bilateral MCAs & $1(4.0)$ \\
Basilar artery + 1 MCA & $1(4.0)$ \\
Mean (SD) maximum diameter of dilated (mm) & \\
Basilar artery & $15.2(8.3)$ \\
Internal carotid artery & $12.7(7.0)$ \\
MCA & $10.1(4.3)$ \\
Thrombus in fusiform/dolichoectatic aneurysm & $7(28.0)$ \\
\hline
\end{tabular}

Note:-DID indicates diffuse intracranial dolichoectasia.

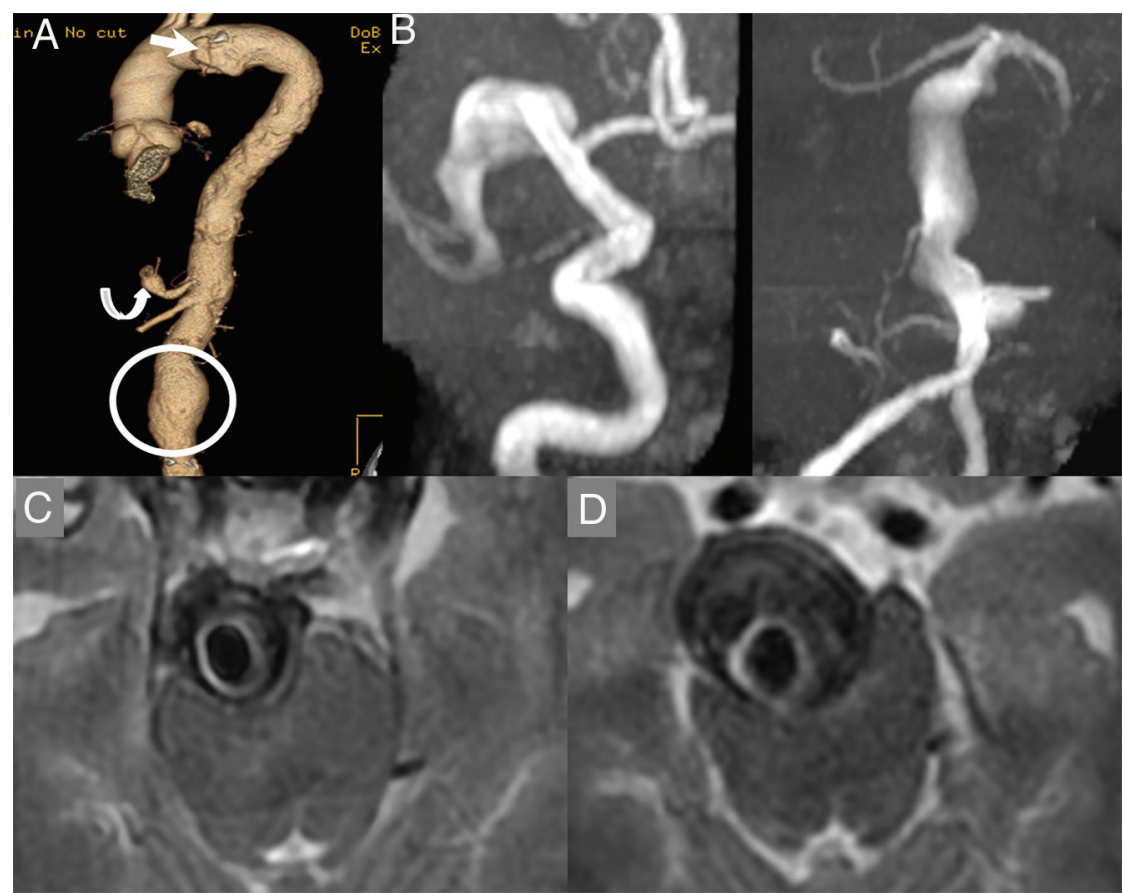

FIG 1. A 67-year-old man who is a former smoker with a history of a penetrating atheromatous ulcer of the aortic arch (white arrow), a 5.6- $\mathrm{cm}$ abdominal aortic aneurysm (circle), and a celiac artery aneurysm (curved arrow, $A$ ). The patient had an episode of dizziness and headache and underwent a noncontrast CT of the head, which demonstrated enlarged intracranial arteries. An MRA demonstrated fusiform aneurysmal dilation of the entire right M1 segment measuring $10 \mathrm{~mm}$ in maximum diameter and a largely thrombosed fusiform aneurysm of the basilar artery, which measured $18 \mathrm{~mm}$ in maximum diameter ( $B$ and $C$ ). Approximately 9 months later, the aneurysm grew to $25 \mathrm{~mm}$ in diameter and started causing obstructive hydrocephalus $(D)$. The patient also had a new perforator pontine infarct at the time (not shown). A programmable ventriculoperitoneal shunt was placed; however, the patient died due to complications of hydrocephalus 3 months later. the overall growth rate was $9.5 \% /$ patient-year. Seven patients (56\%) had imaging evidence of a new infarct after a mean of $60.9 \pm 66.5$ months of follow-up, including 2 patients with brain stem perforator infarcts, 5 patients with cerebellar or thalamic infarcts, and 4 patients with anterior circulation infarcts.

Five patients $(5 / 25,20.0 \%)$ had imaging evidence of subarachnoid hemorrhage at a mean of $59.0 \pm 73.1$ months of follow-up. Of these 5 patients, 3 had rupture of 2 basilar dolichoectatic/ fusiform aneurysms, 1 had rupture of a posterior inferior cerebellar artery dissecting aneurysm, and in 1 patient, the source of the subarachnoid hemorrhage was unclear (On-line Table). Including patients without imaging follow-up, the overall rate of subarachnoid hemorrhage was 5.9\%/year (5 cases over 84.9 patient-years).

The mean clinical follow-up for all 25 patients was $40.8 \pm 62.3$ mass effect from a growing basilar artery fusiform aneurysm, and 1 patient died from intraparenchymal hemorrhage, which was not aneurysm-related. The remaining deaths were noncerebrovascular in nature, including 4 patients who died from cancer and 2 patients who died from unknown causes. In 4 of the 6 patients who had poor neurologic outcome at last follow-up, the cause of the morbidity was related to diffuse intracranial dolichoectasia. Representative case examples are provided in Figs 1-4.

\section{Comparison of Diffuse Intracranial Dolichoectasia and Isolated Vertebrobasilar Dolichoectasia Groups}

A total of 139 patients had vertebrobasilar dolichoectasia with a mean follow-up of $44 \pm 42.9$ months. A case of vertebrobasilar dolichoectasia is demonstrated in Fig 5. None of the patients with vertebrobasilar dolichoectasia progressed to the diffuse form. Patients with diffuse intracranial dolichoectasia were older $(70.9 \pm 14.2$ years versus $60.4 \pm 12.5$ years, $P=.0002$ ), and the age distribution of patients with diffuse intracranial dolichoectasia was older $(P<.0001)$. There was a predominance of males in both patient groups $(84.0 \%$ versus $75.5 \%, P=.51)$. Patients with diffuse intracranial dolichoectasia were significantly more likely to have abdominal aortic aneurysms or ectasias $(62.5 \%$ versus $14.3 \%, P=.01)$ as well as visceral aneurysms $(25.0 \%$ versus $0.0 \%, P<.0001)$. Patients with diffuse intracranial dolichoectasia trended toward higher rates of peripheral artery disease $(16.0 \%$ versus $4.3 \%, P=.07)$ and hypertension $(88.0 \%$ versus $70.3 \%, P=.11)$. Patients with dif- 


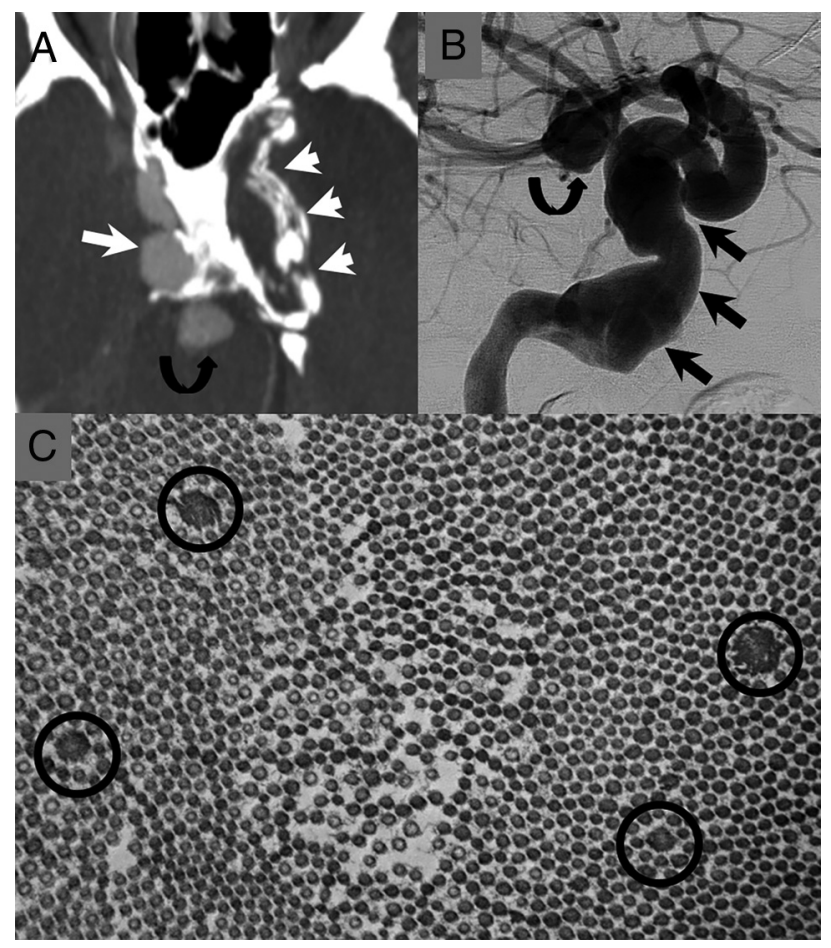

FIG 2. The patient is a 47-year-old man. He presented in his 20 s with 2 large fusiform aneurysms of his cavernous carotid arteries. He later developed an aneurysm of his basilar artery and had a subarachnoid hemorrhage from a dissecting aneurysm of his PICA (not shown). A, CTA image demonstrates a large fusiform aneurysm of the right cavernous carotid artery (long white arrow) and a thrombosed/calcified aneurysm of the left cavernous carotid artery (short white arrows). There is also an aneurysm of the basilar tip (curved black arrow). B, Right ICA cerebral angiogram shows a large fusiform aneurysm of the right petrocavernous carotid artery (straight black arrows) and a basilar tip aneurysm (curved black arrow). C, There was suspicion for underlying connective tissue disease. The patient underwent a skin biopsy. Electron microscopy of the skin biopsy shows multiple abnormally enlarged collagen fibers (black circles) consistent with collagen flowers. These are typically seen in Ehlers-Danlos syndrome. The patient later underwent genetic testing for Loeys-Dietz, Ehlers-Danlos, and Marfan syndromes. The findings of all tests were negative.

fuse intracranial dolichoectasia were significantly more likely to smoke $(68.0 \%$ versus $15.9 \%, P<.0001)$.

Patients with diffuse intracranial dolichoectasia trended toward higher aneurysm growth rates $(46.2 \%$ versus $21.6 \%, P=$ $.09)$ and cerebral infarction rates $(28.0 \%$ versus $13.0 \%, P=.10)$ and were more likely to have SAH on follow-up (20.0\% versus $3.5 \%, P=.007)$. Patients with diffuse intracranial dolichoectasia were less likely to have good neurologic function at follow-up (24.0\% versus $57.6 \%, P=.004)$, were more likely to die $(52.0 \%$ versus $26.6 \%, P=.02$ ), and were more likely to die due to aneurysm-related factors $(24.0 \%$ versus $7.2 \%, P=.02)$. These data are summarized in Table 2.

On multivariate analysis, adjusting for age and smoking history, a diagnosis of diffuse intracranial dolichoectasia was independently associated with higher odds of aneurysm rupture $(\mathrm{OR}=6.33 ; 95 \% \mathrm{CI}, 1.23-35.31 ; P=.02)$ and higher odds of new ischemic stroke $(\mathrm{OR}=18.62 ; 95 \% \mathrm{CI}, 3.63-146.57 ; P=.0003)$. There was no significant difference in the odds of aneurysm growth on multivariate analysis $(\mathrm{OR}=2.23$; 95\% CI, 0.57-8.68; $P=.24)$.

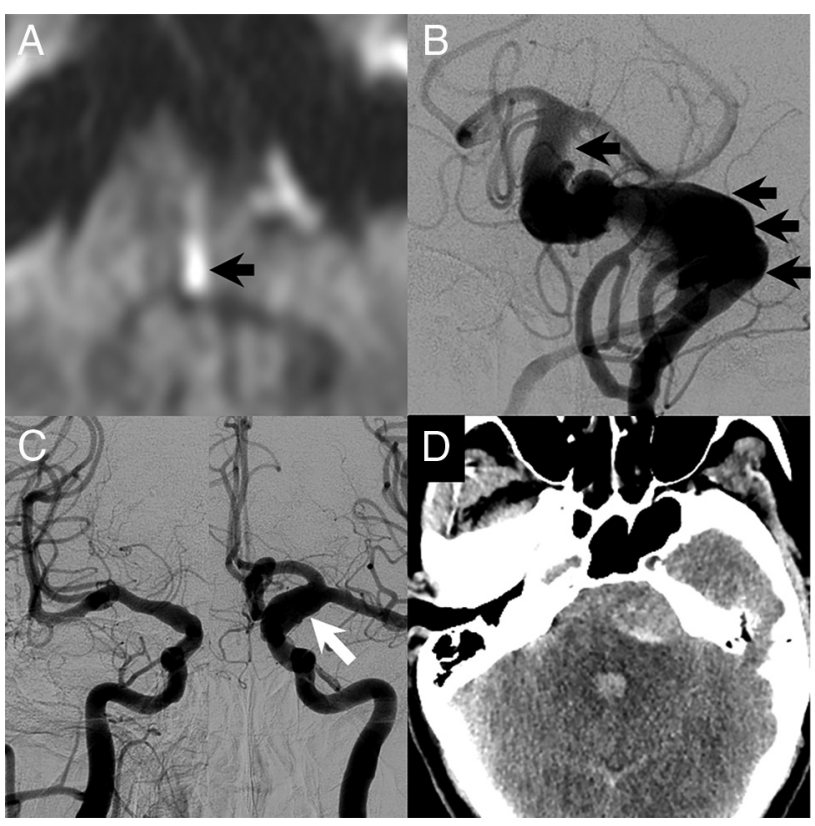

FIG 3. A 51-year-old man with a long history of headaches with associated nausea and vomiting. He had acute-onset left-sided weakness with a prominent left facial droop along with left face, arm, and leg numbness and slurred speech. The patient had no family history of cerebral aneurysms, though his father had an abdominal aortic aneurysm. A, MR imaging at the time of the initial evaluation showed a medial left pontine infarct (black arrow). There was evidence of a large dolichoectatic aneurysm of the basilar artery on MR imaging, and the patient underwent cerebral angiography for further evaluation. $B$, Cerebral angiography demonstrated a fusiform-type aneurysm of the basilar artery with a filling defect that was consistent with thrombus (black arrows). The patient also had diffuse arteriomegaly with dilation of the right supraclinoid ICA to $6 \mathrm{~mm}$ and dilation of the left supraclinoid ICA to $10 \mathrm{~mm}$ (white arrow, C). D, The day following the angiography, the patient had a 10/10 headache. Noncontrast CT at the time showed diffuse subarachnoid hemorrhage with most of the blood products surrounding the basilar artery aneurysm. He died the next day.

\section{DISCUSSION}

Our study of 25 patients with diffuse intracranial dolichoectasia demonstrated a number of notable findings. First, this condition is a relatively rare vascular phenotype, predominantly affecting elderly male smokers with hypertension. The natural history of this disease is dismal, with only a minority surviving with good outcome at a mean follow-up of 3.5 years. Furthermore, among patients with abdominal imaging, $>60 \%$ had abdominal aortic aneurysms or ectasias and nearly $30 \%$ of patients had an additional intracranial saccular aneurysm. Compared with a control group of patients with isolated vertebrobasilar dolichoectasia, patients with diffuse intracranial dolichoectasia were more likely to have aortic and visceral aneurysms, were more likely to smoke, and were more likely to experience aneurysm growth, rupture, and morbidity and mortality on both univariate and multivariate analyses. Overall, these findings suggest that diffuse intracranial dolichoectasia may represent a distinct vascular phenotype from isolated vertebrobasilar dolichoectasia and is likely the manifestation of a diffuse, systemic arteriopathy affecting multiple vascular beds. A summary of our proposed diagnostic criteria for diffuse intracranial dolichoectasia and how they compare with current definitions of vertebrobasilar dolichoectasia is provided in Table 4. 
We fully acknowledge that patients with multivessel intracranial dolichoectasia have been reported previously in the literature. However, prior reports were generally quite small and did not specifically identify multivessel involvement as a distinct entity and did not provide detailed baseline demographic, imaging, and

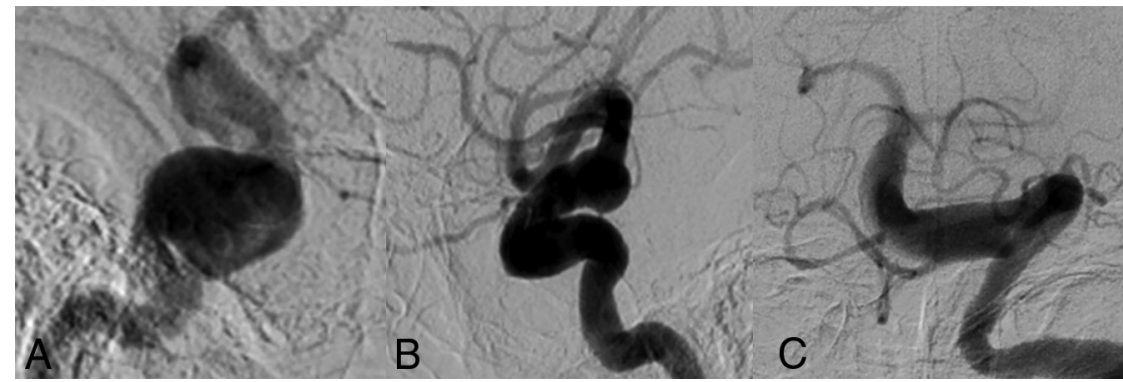

FIG 4. An 87-year-old man with a history of a right third-nerve palsy. A, Right ICA cerebral angiogram demonstrates a $20-\mathrm{mm}$ cavernous carotid fusiform aneurysm with associated dilation of the supraclinoid ICA as well. B, Left ICA cerebral angiogram shows dilation of the left supraclinoid ICA to approximately $10 \mathrm{~mm}$. C, Left vertebral artery cerebral angiogram shows diffuse dilation and tortuosity of the basilar artery measuring $9 \mathrm{~mm}$ in maximum diameter. The cause of the third-nerve palsy was thought to be the right cavernous aneurysm.

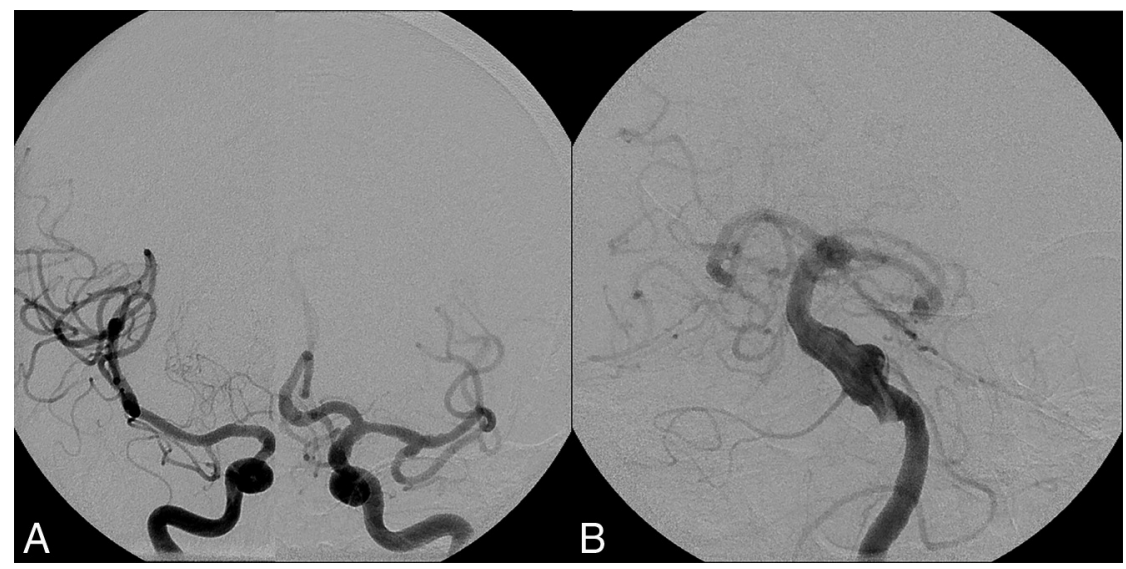

FIG 5. Vertebrobasilar dolichoectasia in a 67-year-old man. A, Right and left ICA cerebral angiograms demonstrate normal-caliber internal carotid arteries, MCAs, and anterior cerebral arteries bilaterally. $B$, Left vertebral artery cerebral angiogram demonstrates an irregular dolichoectatic and fusiform aneurysm involving the entirety of the basilar artery. outcome data to fully characterize our proposed subgroup. In a eview of 9 studies on vertebrobasilar dolichoectasia, Gutierrez noted that approximately $45 \%$ of patients with vertebrobasilar dolichoectasia had some degree of ectasia or dilation of the anterior circulation as well. However, on closer inspection of these studies, it is apparent that the degree of dilation or tortuosity of the anterior circulation and the outcomes of patients with diffuse intracranial dolichoectasia were not reported. ${ }^{3,10,12-14}$ For example, in a case series that our group previously published, 63 of 152 patients (41.5\%) with vertebrobasilar nonsaccular dolichoectatic aneurysms had concomitant dolichoectasias in the anterior circulation by visual inspection. However, only 11 of those patients $(7.2 \%)$ are included in the current study because the other 52 did not meet the size criteria set forth in Table 1 . In fact, even in the large surgical series reported by Anson et $\mathrm{al}^{15}$ and Drake et $\mathrm{al}^{16}$ of patients with surgically treated large fusiform aneurysms, $<5$ percent of cases would meet the diagnostic criteria of diffuse intracranial dolichoectasia. Other small case series and case reports have reported outcomes of patients with diffuse intracranial dolichoectasia with a welldefined underlying etiology such as prior radiation, autosomal dominant polycystic kidney disease, connective tissue disease, or infection. However, findings from these studies do not necessarily apply to the patient population included in our series due to substantial differences in risk factors and etiology. $^{17-23}$

Our comparison of the baseline clini-

Table 4: Comparison of diagnostic criteria and outcomes

\begin{tabular}{|c|c|c|}
\hline & Diffuse Intracranial Dolichoectasia & Vertebrobasilar Dolichoectasia $^{a}$ \\
\hline Imaging appearance & $\begin{array}{l}\text { Fusiform aneurysmal dilation of an entire } \\
\text { vascular segment (ie, supraclinoid ICA, basilar } \\
\text { artery, M1 segment of the MCA) }\end{array}$ & $\begin{array}{l}\text { Fusiform: aneurysmal dilation without definable } \\
\text { neck involving a portion of an arterial segment } \\
\text { with any degree of tortuosity } \\
\text { Dolichoectatic: uniform aneurysmal dilation of an } \\
\text { artery involving the entire basilar or vertebral } \\
\text { or both with any degree of tortuosity } \\
\text { Transitional: uniform aneurysmal dilation of an } \\
\text { artery with superimposed dilation of a portion } \\
\text { of the involved arterial segment }\end{array}$ \\
\hline Distribution & $\begin{array}{l}\geq 2 \text { Intracranial vascular beds (ie, } \\
\quad \text { vertebrobasilar system, left anterior } \\
\quad \text { circulation, or right anterior circulation) }\end{array}$ & Vertebrobasilar system only \\
\hline Size criteria & $\begin{array}{l}\text { Cavernous ICA: } \geq 8.5 \mathrm{~mm} \\
\text { Supraclinoid ICA: } \geq 8.0 \mathrm{~mm} \\
\text { MCA: } \geq 5.0 \mathrm{~mm} \\
\text { Basilar artery: } \geq 6.0 \mathrm{~mm}\end{array}$ & Basilar artery diameter of $>5.0 \mathrm{~mm}$ \\
\hline Growth rate & $10 \% /$ year & $7 \% /$ year 2,10 \\
\hline Ischemic stroke risk & $11 \% /$ year & $3 \% /$ year $^{2,10}$ \\
\hline Aneurysm rupture risk & $6 \% /$ year & $2 \% /$ year $^{2,10}$ \\
\hline
\end{tabular}

${ }^{a}$ Definitions of vertebrobasilar dolichoectasia proposed by Flemming et al. ${ }^{2}$ 
cal characteristics and outcomes of patients with diffuse intracranial dolichoectasia with patients with isolated vertebrobasilar dolichoectasia suggests that at the very least, this entity is a distinctive phenotype or a more diffuse and severe form of vertebrobasilar dolichoectasia. In general, patients with diffuse intracranial dolichoectasia were older, were more likely to have hypertension and other atherosclerotic risk factors, and were more likely to smoke. Furthermore, patients in the diffuse intracranial dolichoectasia group were significantly more likely to have intracranial, abdominal aortic, and visceral arterial aneurysms than those with isolated vertebrobasilar dolichoectasia. These differences are not only isolated to patients studied at our institution. Compared with the systematic review of the literature on vertebrobasilar dolichoectasia published by Gutierrez et al, ${ }^{11}$ patients in our current series were generally older (71 versus 63 years), were more often male ( $84 \%$ versus $64 \%$ ), and were more likely to have hypertension ( $88 \%$ versus $66 \%$ ), smoke ( $68 \%$ versus $42 \%$ ), and have other atherosclerotic risk factors. Furthermore, considering all patients in our series with abdominal imaging, 50\% of patients had abdominal aortic aneurysms compared with an average of $11 \%$ in the vertebrobasilar dolichoectasia literature. ${ }^{11}$ The rate of concomitant saccular aneurysm formation is twice as high as that reported in the literature as well (28\% versus $15 \%)$.

The natural history of patients with diffuse intracranial dolichoectasia was also substantially more aggressive than that of the isolated vertebrobasilar dolichoectasia group as well as that reported in the vertebrobasilar dolichoectasia literature. Twenty percent of patients in our series had subarachnoid hemorrhage on follow-up, for a subarachnoid hemorrhage rate of $6.9 \% /$ patientyear. This compares with rupture rates of just $1 \%-2 \%$ per year reported for dolichoectatic and fusiform aneurysms of both the anterior and posterior circulations. ${ }^{3,4,24}$ The growth rate for patients with diffuse intracranial dolichoectasia was also substantially higher than that reported in the literature among patients with single-vessel dolichoectatic aneurysms (9.5\%/year versus $6.5 \%$ /year). ${ }^{10}$ More than $50 \%$ of the patients in the current series had ischemic stroke after a mean of approximately 5 years of follow-up. Meanwhile, the rates of ischemic stroke among previously published large series of vertebrobasilar dolichoectasia are $3 \%, 11 \%, 16 \%$, and $38 \%$ at $1,5,10$, and 12 years, respectively. ${ }^{11}$

\section{Limitations}

Our study has limitations. First, this is a retrospective study spanning $>15$ years at a single institution. Therefore, there is wide variation in the type of imaging used, clinical management of these patients, and degree of follow-up. In addition, our study is prone to substantial selection bias. Patients who presented with diffuse intracranial dolichoectasia at our tertiary referral institution were more likely to have complications related to this disease, which could explain the poor natural history seen in our study. We do not have access to pathologic specimens or gene samples from the patients included in this study. Thus, we are unable to determine whether the pathologic basis of this disease is similar to that in other fusiform aneurysms and ectasias or whether the degree of vessel wall weakening is, in fact, more severe. While none of the patients in our series had a clinical diagnosis of a genetic connective tissue disease such as Marfan syndrome, Ehlers-Dan- los syndrome, or Loeys-Dietz syndrome, most of the patients included in this series did not receive any genetic testing, so we cannot rule out the presence of an undiagnosed connective tissue disease. Therefore, the total number of patients or the association to secondary diseases may not be as high as that reported in our study. In our comparison of the natural history of diffuse intracranial dolichoectasia with that of vertebrobasilar dolichoectasia, we did not account for the fact that the morphology of the vertebrobasilar dolichoectatic lesions has a significant impact on their natural history. As defined in Table 4, dolichoectatic vertebrobasilar aneurysms have a much more favorable natural history than fusiform or transitional lesions. ${ }^{10}$ Last, because this entity has not been extensively studied outside small case series and case reports, there are no well-defined diagnostic criteria. Our choice of size criteria in diagnosing diffuse intracranial dolichoectasia are somewhat arbitrary (doubling of mean values reported in the literature). Using such strict criteria could have resulted in exclusion of patients with milder or earlier forms of this disease.

\section{CONCLUSIONS}

Our study of 25 patients with diffuse intracranial dolichoectasia demonstrated that this rare disease is associated with a very poor natural history, with about $20 \%$ of patients having subarachnoid hemorrhage on follow-up, $30 \%$ of patients having ischemic stroke, and $25 \%$ of patients with aneurysm-related mass effect. Nearly two-thirds of patients with this disease have abdominal aortic aneurysms, and $25 \%$ have additional saccular intracranial aneurysms. Given the high morbidity and mortality rates associated with this disease and the high rate of concomitant intracranial saccular aneurysm and abdominal aortic aneurysms in this population, these findings suggest that diffuse intracranial dolichoectasia may represent a distinct vascular phenotype. Further research is needed to better characterize the histopathologic and genetic features of diffuse intracranial dolichoectasia and determine ideal treatment options.

Disclosures: Giuseppe Lanzino_UNRELATED: Consultancy: Covidien/Medtronic.* David F. Kallmes—UNRELATED: Consultancy: Minnetronix, ${ }^{*}$ Comments: Data and Safety Monitoring Board; Grants/Grants Pending: Medtronic, MicroVention, Sequent Medical, Surmodics, Codman, NeuroSigma, Comments: preclinical research and clinical trials*; Travel/Accommodations/Meeting Expenses Unrelated to Activities Listed: Medtronic, Comments: FDA panel meeting.* *Money paid to the institution.

\section{REFERENCES}

1. Dispensa BP, Saloner DA, Acevedo-Bolton G, et al. Estimation of fusiform intracranial aneurysm growth by serial magnetic resonance imaging. J Magn Reson Imaging 2007;26:177-83 CrossRef Medline

2. Flemming KD, Wiebers DO, Brown RD Jr, et al. The natural history of radiographically defined vertebrobasilar nonsaccular intracranial aneurysms. Cerebrovasc Dis 2005;20:270-79 CrossRef Medline

3. Passero SG, Rossi S. Natural history of vertebrobasilar dolichoectasia. Neurology 2008;70:66-72 CrossRef Medline

4. Sacho RH, Saliou G, Kostynskyy A, et al. Natural history and outcome after treatment of unruptured intradural fusiform aneurysms. Stroke 2014;45:3251-56 CrossRef Medline

5. Mizutani T. A fatal, chronically growing basilar artery: a new type of dissecting aneurysm. J Neurosurg 1996;84:962-71 CrossRef Medline

6. Mizutani T, Aruga T. "Dolichoectatic" intracranial vertebrobasilar 
dissecting aneurysm. Neurosurgery 1992;31:765-73; discussion 773 CrossRef Medline

7. Mizutani T, Kojima H. Clinicopathological features of non-atherosclerotic cerebral arterial trunk aneurysms. Neuropathology 2000; 20:91-97 CrossRef Medline

8. Mizutani T, Miki Y, Kojima H, et al. Proposed classification of nonatherosclerotic cerebral fusiform and dissecting aneurysms. Neurosurgery 1999;45:253-59; discussion 259-60 CrossRef Medline

9. Mocco J, Huston J, Fargen KM, et al. An angiographic atlas of intracranial arterial diameters associated with cerebral aneurysms. J Neurointerv Surg 2014;6:533-35 CrossRef Medline

10. Nasr DM, Brinjikji W, Rouchaud A, et al. Imaging characteristics of growing and ruptured vertebrobasilar non-saccular and dolichoectatic aneurysms. Stroke 2016;47:106-12 CrossRef Medline

11. Gutierrez J, Sacco RL, Wright CB. Dolichoectasia-an evolving arterial disease. Nat Rev Neurol 2011;7:41-50 CrossRef Medline

12. Passero SG, Calchetti B, Bartalini S. Intracranial bleeding in patients with vertebrobasilar dolichoectasia. Stroke 2005;36:1421-25 CrossRef Medline

13. Yu YL, Moseley IF, Pullicino P, et al. The clinical picture of ectasia of the intracerebral arteries. J Neurol Neurosurg Psychiatry 1982;45: 29-36 CrossRef Medline

14. Nakatomi $\mathrm{H}$, Segawa $\mathrm{H}$, Kurata A, et al. Clinicopathological study of intracranial fusiform and dolichoectatic aneurysms: insight on the mechanism of growth. Stroke 2000;31:896-900 CrossRef Medline

15. Anson JA, Lawton MT, Spetzler RF. Characteristics and surgical treatment of dolichoectatic and fusiform aneurysms. J Neurosurg 1996;84:185-93 CrossRef Medline

16. Drake CG, Peerless SJ. Giant fusiform intracranial aneurysms: re- view of 120 patients treated surgically from 1965 to 1992 . J Neurosurg 1997;87:141-62 CrossRef Medline

17. Schievink WI, Torres VE, Wiebers DO, et al. Intracranial arterial dolichoectasia in autosomal dominant polycystic kidney disease. J Am Soc Nephrol 1997;8:1298-303 Medline

18. Sedat J, Alvarez H, Rodesch G, et al. Multifocal cerebral fusiform aneurysms in children with immune deficiencies report of four cases. Interv Neuroradiol 1999;5:151-56 Medline

19. Naunheim MR, Walcott BP, Nahed BV, et al. Arterial tortuosity syndrome with multiple intracranial aneurysms: a case report. Arch Neurol 2011;68:369-71 CrossRef Medline

20. Azzarelli B, Moore J, Gilmor R, et al. Multiple fusiform intracranial aneurysms following curative radiation therapy for suprasellar germinoma: case report. J Neurosurg 1984;61:1141-45 CrossRef Medline

21. Braunsdorf WE. Fusiform aneurysm of basilar artery and ectatic internal carotid arteries associated with glycogenosis type 2 (Pompe's disease). Neurosurgery 1987;21:748-49 CrossRef Medline

22. Capone PM, Mechtler LL, Bates VE, et al. Multiple giant intracranial aneurysms associated with lymphomatoid granulomatosis: a magnetic resonance imaging and angiographic study. J Neuroimaging 1994;4:109-11 CrossRef Medline

23. Siqueira Neto JI, Silva GS, De Castro JD, et al. Neurofibromatosis associated with moyamoya arteriopathy and fusiform aneurysm: case report [in Portuguese]. Arq Neuropsiquiatr 1998;56:819-23 CrossRef Medline

24. Flemming KD, Wiebers DO, Brown RD Jr, et al. Prospective risk of hemorrhage in patients with vertebrobasilar nonsaccular intracranial aneurysm. J Neurosurg 2004;101:82-87 CrossRef Medline 